\title{
Dielectric Characteristics Studies of Esters (Amyl Acetate, Isoamyl Acetate) with Solvents (Carbon Tetrachloride, Benzene, Acetone)
}

\author{
R. ANANDHI and P. KRISHNAMURTHI
}

Department of Physics, Varuvan Vadivelan Institute of Technology, Dharmapuri, Tamilnadu- 636 703, India

drkrishnamurthi@gmail.com

Received 6 May 2015 / Accepted 18 May 2015

\begin{abstract}
The Kirkwood correlation factor (g) of binary mixtures of amyl acetate and isoamyl acetate with in benzene, carbon tetrachloride and acetone were obtained from a measurement of dielectric constant, density and refractive index at $301 \mathrm{~K}$. The fluid structure of amyl acetate and isoamyl acetate is discussed. In amyl acetate and isoamyl acetate in carbon tetrachloride, benzene and acetone, a dimeric linear chain with the individual dipoles more or less parallel orientation is preferred. In amyl acetate, with increase of concentration, the value of $g$ tending to unity indicates the parallel orientation.
\end{abstract}

Keywords: Amyl acetate, Kirkwood correlation factor, Dielectric constant, Refractive index

\section{Introduction}

The studies of molecular interactions interesting one to provide valuable information regarding dipole-dipole, dipole-induced dipole interactions and these in turn help to understand the molecular structure of the polar solutes ${ }^{1-5}$.

The Kirkwood theory ${ }^{6-8}$ of dipolar liquids defines the short range dipolar interactions through a dimensionless parameter g. Kirkwood correlation factor of dielectric polarization is a measure of the short range intermolecular forces that leads to dipole orientation in solutions. The $\mathrm{g}$ factor varies with concentration, temperature and solute environment done by many authors ${ }^{9-17}$. Fluid structure detected by a measurement of concentration dependence of Kirkwood correlation factor for the esters. An increase in $\mathrm{g}$ is expected on linear or cyclic. The clarification of the structure of chains in a solvent environment is very important in the context of biological systems. Hence we have taken up the study of Kirkwood correlation factor and the related parameters of two amyl acetate and isoamyl acetate substitutes in the solution state in solvent carbon tetrachloride, benzene and acetone.

\section{Experimental}

Dielectric constant and refractive index was measured using Toshniwal RL09 type dipole meter at $300 \mathrm{KHz}$ with accuracy of \pm 0.0002 and Abbe's refractometer with accuracy of 
\pm 0.002 for sodium $D$-line respectively at temperature $301 \mathrm{~K}$. The densities were obtained by a $25 \mathrm{ml}$ specific gravity bottle with accuracy of $0.0001 \mathrm{gcm}^{-3}$. The solutes and solvents of AR grade were used without further purification having $99 \%$ of purity. The high dielectric permittivity taken as $\varepsilon_{\infty}$ due to atomic polarization over electronic polarization of the solute.

\section{THEORY}

\section{Limiting Polarization method (PM)}

The polarization of the solution $\mathrm{P}_{12}$ is find form the dielectric measurements is

$$
\mathrm{P}_{12}=\left[\frac{\varepsilon_{12}-1}{\varepsilon_{12}+2}\right]\left[\frac{\mathrm{m}_{1} \mathrm{x}_{1}+\mathrm{m}_{2} \mathrm{x}_{2}}{\mathrm{~d}_{12}}\right]
$$

$\varepsilon_{12}$ is the dielectric constant of the solution, $\mathrm{m} 1$ and $\mathrm{m} 2$ are the molecular weight of the component, $\mathrm{d}_{12}$ is the density of the component $\mathrm{P}_{12}$ is the sum of the polarization contributions of the two components.

$$
\mathrm{P}_{12}=\mathrm{P}_{1} \mathrm{X}_{1}+\mathrm{P}_{2} \mathrm{X}_{2}
$$

Where $\mathrm{P}_{1}$ and $\mathrm{P}_{2}$ are the molar polarization of the solvent and solute respectively. $\mathrm{P}_{2}$ is determined using the above relation $\mathrm{P}_{2}$ plotted against $\mathrm{x}_{2}$ and the resultant curve may be extrapolated to $X_{2}=0$ to obtain the polarization at infinite dilution $\mathrm{P}_{2} \infty$. This value of $\mathrm{P}_{2} \infty$ includes the contribution of electronic and atomic polarizations also which taken into consideration gives the orientation polarization of the solute molecule and thereby the value of the dipole moment from the following relation.

$$
\mu_{2}=\left[\frac{9 \mathrm{KT}}{4 \pi \mathrm{N}} \mathrm{P}_{2} \mu \mathrm{T}\right]^{1 / 2}
$$

Where $P_{2} \mu=P_{2} \infty-R_{D}$ and $R_{D}$ is the molar refraction.

$$
\mathrm{R}_{\mathrm{D}}=\left[\frac{\mathrm{n}_{2}^{2}-1}{\mathrm{n}_{2}^{2}+2}\right]\left[\frac{\mathrm{m}_{2}}{\mathrm{~d}_{2}}\right]
$$

Insertion of the appropriate values for the constants leads to

$$
\mu_{2}=0.01281 \times 10^{-18}\left[\mathrm{P}_{2} \mu \mathrm{T}\right]^{1 / 2}
$$

\section{Kirkwood correlation factor}

The Kirkwood-Froehlich correlation factor of dielectric polarization $\mathrm{g}$ is an important parameter which provides some insight into short range intermolecular forces that arise due to dipole-dipole interactions and hence gives some information regarding local ordering of polar solute molecules in solutions. By considering each kind of multimers as a separate entity and assuming that the total dipole moment of the cluster of one type multimers has no specific correlation with that of the other types, the extended Kirkwood-Froehlich's expression for solutions can be written as for pure liquids and solution.

The structural information about the liquids from the dielectric parameters may be obtained using the Kirkwood correlation factor $\mathrm{g}^{\mathrm{f}}$ this factor is also a parameter for obtaining information regarding orientation of electric dipoles in polar liquids. The $\mathrm{g}$ for pure liquid can be obtained from the following expression. 


$$
\frac{4 \pi N \mu^{2} \rho}{9 K T M} g=\frac{\left(\varepsilon_{0}-\varepsilon_{\infty}\right)\left(2 \varepsilon_{0}+\varepsilon_{\infty}\right)}{\varepsilon_{0}\left(\varepsilon_{\infty}+2\right)^{2}}
$$

A modified form of this equation has been used to study the orientation of electric dipoles in binary mixtures. For the mixtures of two polar liquids, say 1 and 2, equation (1) is modified by assuming that $\mathrm{g}^{\text {eff }}$ has become the effective correlation factor in the mixture. The Kirkwood equation for the mixture can be expressed as follows

$$
\frac{4 \pi N}{9 K T}\left(\frac{\mu_{1}^{2} \rho_{1}}{M_{1}} X_{1}+\frac{\mu_{2}^{2} \rho_{2}}{M_{2}} X_{2}\right) g^{\text {eff }}=\frac{\left(\varepsilon_{12}-\varepsilon_{\infty 12}\right)\left(2 \varepsilon_{12}+\varepsilon_{\infty 12}\right)}{\varepsilon_{12}\left(\varepsilon_{\infty 12}+2\right)^{2}}
$$

Where $\mathrm{g}^{\text {eff }}$ is the effective Kirkwood correlation factor for a binary mixture with $\mathrm{X}_{1}$ and $\mathrm{X}_{2}$ as mole fractions of liquids 1 and 2 respectively. $\mathrm{N}$ is Avogadro's number, where $\varepsilon_{12}$ represents the dielectric constant of the components, $\varepsilon_{\infty}$ is the high dielectric Permittivity, Due to induced polarization of the polar component in the pure solute and finally $\mu_{\mathrm{i}}$ is the dipole moment components.

We also assume that correlation factors of solute molecules are affected by the same amount $\mathrm{g}^{\mathrm{f}}$ in the mixture. The Kirkwood-Frohlich equation for the mixture can be written as

$$
\frac{4 \pi N}{9 K T}\left(\frac{\mu_{1}^{2} \rho_{1} g_{1}}{M_{1}} X_{1}+\frac{\mu_{2}^{2} \rho_{2} g_{2}}{M_{2}} X_{2}\right) g^{f}=\frac{\left(\varepsilon_{12}-\varepsilon_{\infty 12}\right)\left(2 \varepsilon_{12}+\varepsilon_{\infty 12}\right)}{\varepsilon_{12}\left(\varepsilon_{\infty 12}+2\right)^{2}}
$$

The Kirkwood correlation factors $\mathrm{g}_{1}, \mathrm{~g}_{2}$ for individual species are modified by assuming for solute-solvent mixture that two kinds of intermolecular hydrogen bonds exist. One is the hydrogen bond between the solute-solvent molecules. The plots of $g^{f} v s . x_{2}$ are shown in Figure 3 and 4.

Excess permittivity $\left(\varepsilon^{E}\right)$

For the detection of the cooperative domains in the mixture it is useful to compute the excess permittivity $\varepsilon^{E}$ which may be further used to study dynamics of the mixture due to molecular interactions. The excess permittivity $\varepsilon^{E}$ is defined as

$$
\varepsilon^{E}=\left(\varepsilon_{12}-n_{12}^{2}\right)-\left[\left(\varepsilon_{1}-n_{1}^{2}\right) \mathrm{x}_{1}+\left(\varepsilon_{2}-n_{2}^{2}\right) \mathrm{x}_{2}\right]
$$

Where $\mathrm{X}$ - mole fraction and suffices 12, 1, 2 represents mixture, liquid 1 and liquid 2 respectively. The excess permittivity provides qualitative information about structural aspects of the mixture.

If $\varepsilon^{E}=0$ indicates the liquid 1 and 2 do not interact at all. If $\varepsilon^{E}<0$ indicates the liquid 1 and 2 interaction in such a way that the total effective dipole gets reduced. This suggests that the liquid 1 and 2 may form multimers leading to less effective dipoles or in other words there is a tendency of dipole to align in antiparallel direction. If $\varepsilon^{E}>0$ indicates the liquid 1 and 2 interaction in such a way that the total effective dipole moment increases. There is a tendency of dipole aligned in parallel direction.

\section{Results and Discussion}

The experimental values of dielectric constant $\left(\varepsilon_{12}\right)$, density $\left(\mathrm{kg} / \mathrm{m}^{3}\right)$, refractive index $\left(\mathrm{n}_{12}\right)$, are given in Table 1 to 6 also given the predicted values of polarization $\left(\mathrm{P}_{12}\right)$ and linear correlation factor $\left(\mathrm{g}{ }^{\text {eff }}\right.$ ) with the concentration of amyl acetate and isoamyl acetate with 
carbon tetrachloride, benzene and acetone at $301 \mathrm{~K}$. The dipole moments were determined from the experimental values by the method of polarization given Table 7 .

Table 1. Amyl acetate with carbon tetrachloride

\begin{tabular}{cccccc}
\hline $\mathrm{X}_{2}$ & $\varepsilon_{12}$ & $\rho_{12} \mathrm{~kg} / \mathrm{m}^{3}$ & $\mathrm{n}_{12}$ & $\mathrm{P}_{12}$ & $\mathrm{~g}^{\text {eff }}$ \\
\hline 0.017 & 2.358 & 1.586 & 1.469 & 30.130 & 3.972 \\
0.026 & 2.387 & 1.579 & 1.468 & 30.660 & 3.062 \\
0.034 & 2.416 & 1.572 & 1.468 & 31.190 & 2.574 \\
0.043 & 2.445 & 1.565 & 1.467 & 31.720 & 2.305 \\
0.051 & 2.474 & 1.557 & 1.466 & 32.240 & 2.125 \\
0.06 & 2.503 & 1.550 & 1.466 & 32.760 & 1.977 \\
0.077 & 2.561 & 1.536 & 1.465 & 33.800 & 1.792 \\
0.086 & 2.590 & 1.529 & 1.464 & 34.310 & 1.733 \\
0.103 & 2.648 & 1.515 & 1.463 & 35.340 & 1.632 \\
0.157 & 2.822 & 1.472 & 1.459 & 38.390 & 1.466 \\
0.175 & 2.880 & 1.458 & 1.458 & 39.390 & 1.429 \\
0.22 & 3.025 & 1.423 & 1.455 & 41.900 & 1.362 \\
0.266 & 3.170 & 1.387 & 1.452 & 44.400 & 1.316 \\
\hline
\end{tabular}

Table 2. Amyl acetate with benzene system

\begin{tabular}{cccccc}
\hline $\mathrm{x}_{2}$ & $\varepsilon_{12}$ & $\rho_{12}$ & $\mathrm{n}_{12}$ & $\mathrm{P}_{12}$ & $\mathrm{~g}^{\text {eff }}$ \\
\hline 0.033 & 2.417 & 0.870 & 1.508 & 29.17 & 2.706 \\
0.049 & 2.445 & 0.871 & 1.507 & 29.75 & 2.195 \\
0.065 & 2.474 & 0.871 & 1.506 & 30.33 & 1.938 \\
0.081 & 2.502 & 0.871 & 1.505 & 30.92 & 1.783 \\
0.096 & 2.530 & 0.871 & 1.504 & 31.50 & 1.678 \\
0.111 & 2.559 & 0.871 & 1.503 & 32.08 & 1.603 \\
0.142 & 2.616 & 0.872 & 1.501 & 33.24 & 1.501 \\
0.156 & 2.644 & 0.872 & 1.500 & 33.82 & 1.465 \\
0.185 & 2.701 & 0.872 & 1.498 & 34.99 & 1.409 \\
0.268 & 2.871 & 0.874 & 1.492 & 38.47 & 1.312 \\
0.294 & 2.928 & 0.874 & 1.490 & 39.63 & 1.291 \\
0.357 & 3.070 & 0.875 & 1.485 & 42.52 & 1.252 \\
0.417 & 3.212 & 0.876 & 1.480 & 45.41 & 1.225 \\
\hline
\end{tabular}

Table 3. Amyl acetate with acetone system

\begin{tabular}{cccccc}
\hline $\mathrm{x}_{2}$ & $\varepsilon_{12}$ & $\rho_{12}$ & $\mathrm{n}_{12}$ & $\mathrm{P}_{12}$ & $\mathrm{~g}^{\text {eff }}$ \\
\hline 0.044 & 21.664 & 0.802 & 1.361 & 64.82 & 1.241 \\
0.065 & 21.496 & 0.803 & 1.362 & 65.47 & 1.241 \\
0.085 & 21.328 & 0.804 & 1.362 & 66.11 & 1.240 \\
0.106 & 21.160 & 0.805 & 1.363 & 66.74 & 1.239 \\
0.125 & 20.992 & 0.805 & 1.363 & 67.37 & 1.238 \\
0.144 & 20.824 & 0.806 & 1.364 & 68.00 & 1.237 \\
0.181 & 20.488 & 0.808 & 1.365 & 69.24 & 1.236 \\
0.199 & 20.320 & 0.809 & 1.365 & 69.86 & 1.235 \\
0.234 & 19.984 & 0.811 & 1.366 & 71.07 & 1.234 \\
0.330 & 18.976 & 0.816 & 1.369 & 74.61 & 1.229 \\
0.359 & 18.640 & 0.818 & 1.370 & 75.75 & 1.227 \\
0.428 & 17.800 & 0.823 & 1.373 & 78.51 & 1.224 \\
0.490 & 16.960 & 0.827 & 1.375 & 81.14 & 1.220 \\
\hline
\end{tabular}


Table 4. Isoamyl acetate with carbon tetrachloride system

\begin{tabular}{cccccc}
\hline $\mathrm{x}_{2}$ & $\mathcal{E}_{12}$ & $\rho_{12}$ & $\mathrm{n}_{12}$ & $\mathrm{P}_{12}$ & $\mathrm{~g}^{\text {eff }}$ \\
\hline 0.017 & 2.372 & 1.599 & 1.469 & 30.10 & 4.242 \\
0.026 & 2.408 & 1.599 & 1.469 & 30.60 & 3.288 \\
0.034 & 2.444 & 1.598 & 1.468 & 31.09 & 2.840 \\
0.043 & 2.480 & 1.598 & 1.468 & 31.57 & 2.544 \\
0.051 & 2.516 & 1.597 & 1.468 & 32.04 & 2.344 \\
0.060 & 2.552 & 1.597 & 1.467 & 32.50 & 2.219 \\
0.077 & 2.624 & 1.596 & 1.466 & 33.39 & 2.035 \\
0.086 & 2.660 & 1.595 & 1.466 & 33.83 & 1.962 \\
0.103 & 2.732 & 1.594 & 1.465 & 34.67 & 1.863 \\
0.157 & 2.948 & 1.591 & 1.463 & 37.01 & 1.680 \\
0.175 & 3.020 & 1.590 & 1.462 & 37.73 & 1.644 \\
0.220 & 3.200 & 1.588 & 1.460 & 39.42 & 1.572 \\
0.266 & 3.380 & 1.585 & 1.458 & 40.95 & 1.521 \\
\hline
\end{tabular}

Table 5. Isoamyl acetate with benzene system

\begin{tabular}{cccccc}
\hline $\mathrm{x}_{2}$ & $\varepsilon_{12}$ & $\rho_{12}$ & $\mathrm{n}_{12}$ & $\mathrm{P}_{12}$ & $\mathrm{~g}^{\text {eff }}$ \\
\hline 0.033 & 2.431 & 0.884 & 1.508 & 28.93 & 2.942 \\
0.049 & 2.466 & 0.890 & 1.508 & 29.37 & 2.429 \\
0.065 & 2.502 & 0.897 & 1.507 & 29.82 & 2.170 \\
0.081 & 2.537 & 0.904 & 1.506 & 30.25 & 2.013 \\
0.096 & 2.572 & 0.911 & 1.505 & 30.67 & 1.906 \\
0.111 & 2.608 & 0.918 & 1.504 & 31.09 & 1.829 \\
0.142 & 2.679 & 0.931 & 1.503 & 31.90 & 1.724 \\
0.156 & 2.714 & 0.938 & 1.502 & 32.30 & 1.686 \\
0.185 & 2.785 & 0.952 & 1.500 & 33.07 & 1.627 \\
0.268 & 2.997 & 0.992 & 1.496 & 35.23 & 1.522 \\
0.294 & 3.068 & 1.006 & 1.494 & 35.91 & 1.499 \\
0.357 & 3.245 & 1.040 & 1.490 & 37.51 & 1.455 \\
0.417 & 3.422 & 1.074 & 1.486 & 38.99 & 1.423 \\
\hline
\end{tabular}

Table 6. Isoamyl acetate with acetone system

\begin{tabular}{cccccc}
\hline $\mathrm{x}_{2}$ & $\varepsilon_{12}$ & $\rho_{12}$ & $\mathrm{n}_{12}$ & $\mathrm{P}_{12}$ & $\mathrm{~g}^{\text {eff }}$ \\
\hline 0.044 & 21.678 & 0.815 & 1.361 & 63.78 & 1.242 \\
0.065 & 21.517 & 0.823 & 1.362 & 63.90 & 1.241 \\
0.085 & 21.356 & 0.830 & 1.363 & 64.02 & 1.240 \\
0.106 & 21.195 & 0.838 & 1.364 & 64.13 & 1.239 \\
0.125 & 21.034 & 0.845 & 1.364 & 64.24 & 1.239 \\
0.144 & 20.873 & 0.853 & 1.365 & 64.34 & 1.238 \\
0.181 & 20.551 & 0.868 & 1.366 & 64.53 & 1.237 \\
0.199 & 20.390 & 0.875 & 1.367 & 64.62 & 1.236 \\
0.234 & 20.068 & 0.890 & 1.368 & 64.79 & 1.235 \\
0.330 & 19.102 & 0.935 & 1.373 & 65.20 & 1.231 \\
0.359 & 18.780 & 0.950 & 1.374 & 65.30 & 1.230 \\
0.428 & 17.975 & 0.988 & 1.378 & 65.50 & 1.227 \\
0.490 & 17.170 & 1.025 & 1.381 & 65.60 & 1.225 \\
\hline
\end{tabular}


Table 7. Dipole moments of solute with solvent systems

\begin{tabular}{ccccc}
\hline S.No. & Solute & Solvent & $\mathrm{P}_{2} \mu$ & $\mu$ \\
\hline \multirow{3}{*}{1} & \multirow{3}{*}{ Amyl acetate } & $\mathrm{CCl}_{4}$ & 29 & 2.48 \\
& & Benzene & 17 & 2.48 \\
& & Acetone & 94 & 1.98 \\
2 & $\mathrm{CCl}_{4}$ & 47 & 2.48 \\
2 & \multirow{2}{*}{ Isoamyl acetate } & Benzene & 37 & 2.43 \\
& & Acetone & 37 & 1.13 \\
\hline
\end{tabular}

From the Figure 1 and 2 for the mixture of carbon tetrachloride, benzene, polarization decreases but acetone mixture increases. Polarization variation depends on the polarization of pure liquids. Acetone is more polarization in the pure states so increasing the concentration acetone than increasing the polarization, but in carbon tetrachloride and benzene smoothly decrease. It is solute-solvent interaction taking place. From the polarization at infinite dilution $\mathrm{P} 2 \infty$ and $\mathrm{R}_{\mathrm{D}}$ find the value obtained $\mathrm{P}_{2} \mu$. From this value dipole moment of the pure liquids are given Table 7 with literature value.

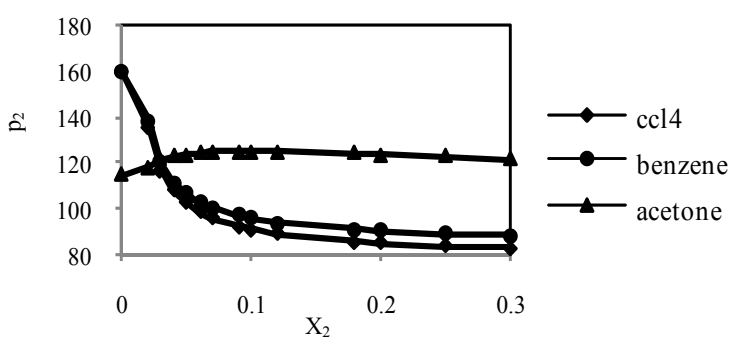

Figure 1. Plots $\mathrm{P}_{2}$ with the mole fraction of amyl acetate

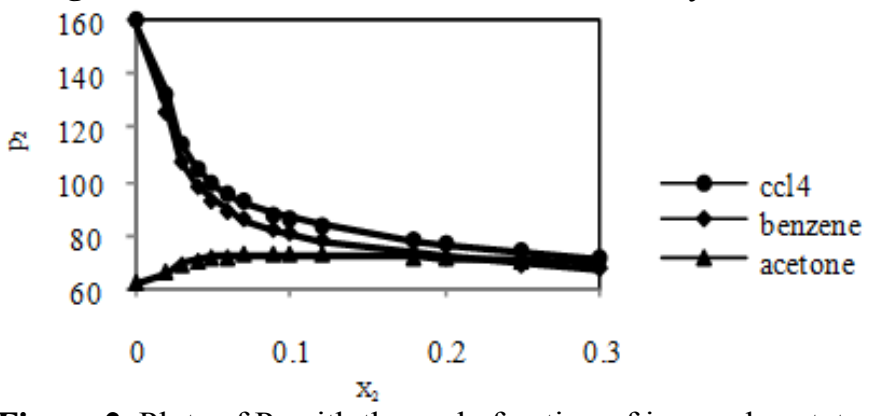

Figure 2. Plots of $\mathrm{P}_{2}$ with the mole fraction of isoamyl acetate

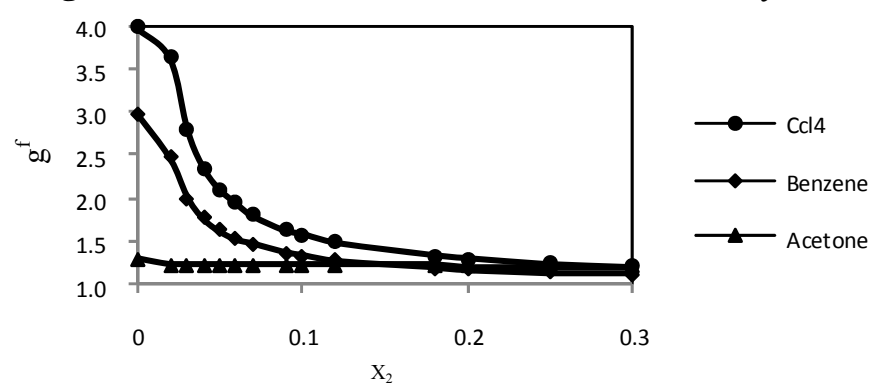

Figure 3. Plots $g^{f}$ with the mole fraction of amyl acetate 


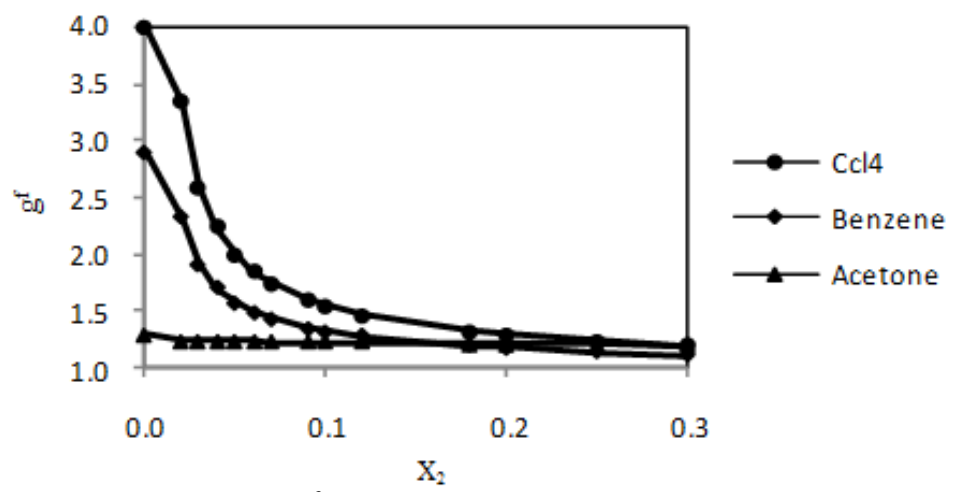

Figure 4. Plots of $\mathrm{g}^{\mathrm{f}}$ with the mole fraction of isoamyl acetate

The value of $g$ is directly related to the correlation between the directions of a particular molecule under consideration with the molecules in its neighborhood in solution. The dipoles whose dipole-dipole interaction lead to a positive correlation, parallel orientation gives $\mathrm{g}>1$ are called $\alpha$-multimers. The dipoles whose dipole-dipole interaction lead to a negative correlation, anti-parallel orientation gives $\mathrm{g}<1$ are called $\beta$ - multimers.

From Figure 1 and 2, the Kirkwood correlation factor of dielectric polarization of amyl acetate and isoamyl acetate in carbon tetrachloride, benzene gradually increases with increasing concentration of the solute which clearly indicates more and more $\beta$-multimers get converted into $\alpha$-multimers. This behaviour can be attributed to the interaction of the lone pair electrons with the solvent or hyper conjugation effect of the acetate group which is present in solution.

The amyl acetate and isoamyl acetate with an acetone investigated. It can be observed from the Figure 1 and 2; the $g$ values are almost around unity which clearly indicates the strong of solute-solute interactions and also solute-solvent interactions and the idealistic behaviour of polar solvent.

The Figure 5 showed the plots of $\varepsilon^{E}$ for the binary mixture of amyl acetate and isoamyl acetate with carbon tetrachloride, benzene and acetone. In the solvent benzene $\varepsilon^{E}$ is negative. It is total effective dipole get reduced. The liquid mixture may for multimer leads to less effective dipole or dipole to align anti parallel orientation. In the solvent carbon tetrachloride and acetone $\varepsilon^{E}$ is positive. It is total effective dipole get increased then increasing dipole moment then aligned dipole in parallel orientation. As similar results observe in the case of isoamyl acetate as shown in Figure 6. The order of solute solvent interactions are benzene $<$ carbon tetrachloride $<$ benzene.

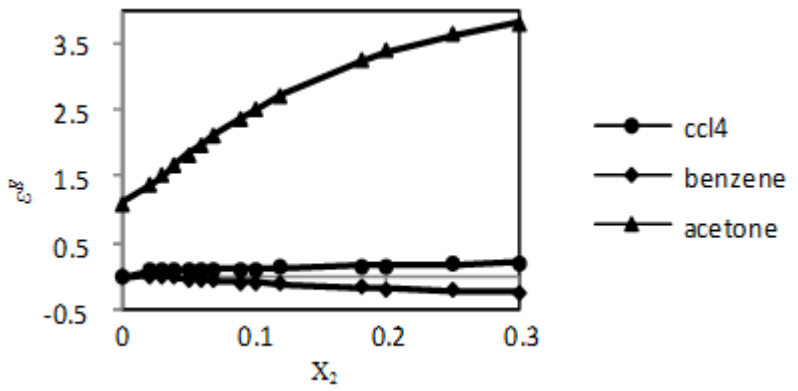

Figure 5. Plots of $\varepsilon^{E}$ with the mole fraction of amyl acetate 


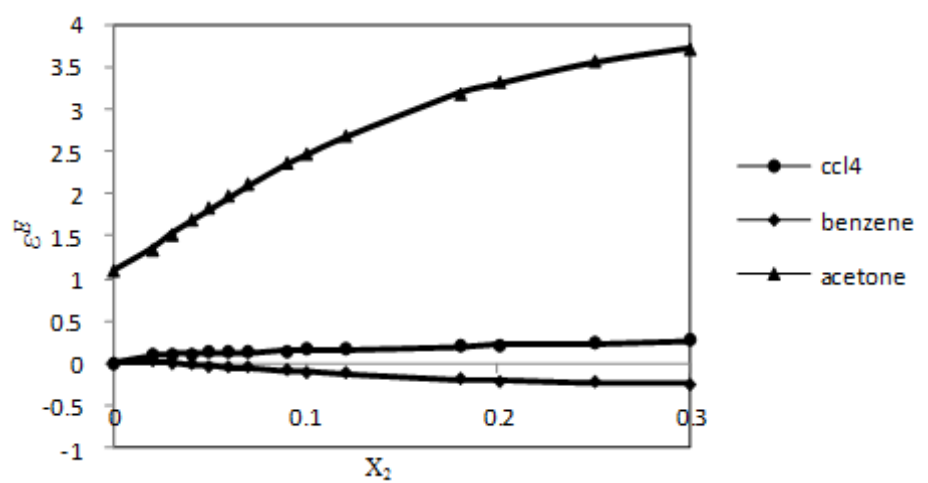

Figure 6. Plots of $\varepsilon^{E}$ with the mole fraction of isoamyl acetate

\section{Conclusion}

The polarization of the solution, Kirkwood correlation factors of dielectric polarization and excess permittivity of two acetates dissolved in carbon tetrachloride, benzene and acetone of various concentrations have been reported. The gradually increasing value of $g$ with the increasing concentration of solute in the solvent clearly indicates more $g^{\text {eff }}$ variation. Different $\mathrm{g}^{\text {eff }}$ values for amyl acetate and isoamyl acetate in carbon tetrachloride, benzene attributed to the idealistic behavior of the solvent and two acetates in acetone attributed to the non idealistic behavior of the solvent. From the polarization, linear correlation factor and excess permittivity is in the order of benzene $<$ carbon tetrachloride $<$ acetone amyl acetate / isoamyl acetate as one of the components.

\section{References}

1. Thenappan T and Subramainan M, Indian J Pure Appl Phys., 2001, 39, 694-697.

2. Tripathy S and Swain B B, Chem Papers, 1991, 45(3), 321- 329.

3. Malathi M, Sabesan R and Krishnan S, Pramana J Phys., 2005, 65(3), 529-534; DOI:10.1007/BF02704211

4. Thenappan T and Subramanian M, Indian J Pure Appl Phys., 2001, 39(11), 694-697.

5. Thenappan T and Sankar U, Indian J Pure Appl Phys., 2004, 42(6), 435-439.

6. $\quad$ Kirkwood J G, J Chem Phys., 1939, 7(10), 911-919; DOI:10.1063/1.1750343

7. Frohlich H, J Chem Soc., 1966, 68, 2036.

8. Frohlich H, Theory of dielectrics (Clarendon Press, Oxford, 1958)

9. Sabesan R, Varadrajan R R and Sargurumoorthy M, Adv Mol Relax Interact Processes, 1981, 20(3), 137-148; DOI:10.1016/0378-4487(81)80010-6

10. Bottcher C J F, Theroy of dielectric polarization (Elsevier, Amsterdam, 1973).

11. Smyth C P, In Molecular Interactions Edited by Ratajczak and Orville-Thomas W J (John Wiley and Sons NY1980).

12. Mizushima M, Shimanouchi T, Nagakura S, Kuratani K, Tsuboi M, Baba H and Fujioka O, J Am Chem Soc., 1950, 72(8), 3490-3494; DOI:10.1021/ja01164a048

13. Ali A and Nain A K, Acoustic Lett., 1996, 19, 18.

14. Ali A and Nain A K, Indian J Pure Appl Phys., 1997, 35, 729-733.

15. Vir Singh Rangra V A and Sharma D R, Indian J Pure Appl Phys., 2003, 41, 630-633.

16. Vyas A D, Raju G S and Das Anitha et al., Indian J Pure Appl Phys., 2002, 40, 69.

17. Anwar Ali, Indian J Pure Appl Phys., 2001, 39, 421. 